\title{
SIMULATION OF ELASTIC-DISSIPATIVE CONNECTION OF MULTI-AXLE BLOCK-MODULAR AGRICULTURAL TRACTOR MODULES
}

\author{
Volodymyr Bulgakov $^{1}$, Volodymyr Kuvachov ${ }^{2}$, Semjons Ivanovs ${ }^{3}$, Viktor Melnyk ${ }^{4}$ \\ ${ }^{1}$ National University of Life and Environmental Sciences of Ukraine, Ukraine; \\ ${ }^{2}$ Tavria State Agrotechnological University, Ukraine; \\ ${ }^{3}$ Latvia University of Life Sciences and Technologies, Latvia; \\ ${ }^{4}$ Kharkiv Petro Vasylenko National Technical University of Agriculture, Ukraine \\ semjons@apollo.lv, viktor_melnik@ukr.net
}

\begin{abstract}
Creation of a tractor according to a modular scheme, by combining the power and the technological modules into a single multi-axle power unit can essentially improve its traction and coupling properties. Research in possibilities how to increase smooth running of a multi-axle block-modular tractor by damping its vertical oscillations due to throttling of the hydraulic system of the rear suspension mechanism of the power module is an urgent task. Synthesis of the connection parameters of the modules of a multi-axle block-modular agricultural tractor was effected by simulating the conditions of its operation on a PC. The model of functioning of a hydraulic suspension mechanism of the power module of a multi-axle block-modular agricultural tractor with a sudden narrowing of the main hydraulic line of the main hydraulic cylinder, as a throttling system, was represented by a combination of elastic and dissipative elements. Creation of a dynamic system with viscoelastic damping by throttling the hydraulic system of the suspension mechanism of the power module allows damping the oscillations of block-modular units in the vertical plane in a wider frequency band $\left(0 \ldots 16 \mathrm{~s}^{-1}\right)$. In addition, dispersion of the mutual vertical oscillations of the power and the technological modules during the agricultural operations is reduced by more than 3 times, and the allowed vibration load of the operator is ensured.
\end{abstract}

Keywords: block tractor, module, oscillations, damping.

\section{Introduction}

The development of tractors has a clear tendency to increase the engine power [1]. However, it is not always possible to proportionally increase the productivity of aggregated agricultural machines [2]. The prospects to increase the efficiency of the machine-and-tractor aggregates, to reduce their specific fuel consumption, etc. on the basis of the classical methods of use are to a great extent exhausted [3-5]. This problem can be solved by implementing the traction-energy concept of the development of the energy facilities on the basis of their block-modular structure [6;7].

Application of agricultural aggregates, based on a multi-axle block-modular agricultural tractor, makes it possible to ensure practically the entire scope of agricultural operations in a wide range of tractive efforts, and, first of all, to perform the basic tillage operations [7;8]. Due to their sufficiently high versatility, the multi-axle block-modular agricultural tractors can be aggregated with almost all trailed, semi-mounted and mounted agricultural machines and implements (Fig. 1). The main devices that protect the energy and the technological modules of a multi-axle block-modular agricultural tractor from the dynamic effects of irregularities in the profile of agricultural backgrounds are the suspension and the tires. However, its performance in general-purpose technological operations leads to the fact that the standard suspension system cannot provide regulation of the elastic and the damping characteristics [9], which leads to a high amplitude of forward vertical oscillations of the aggregate. And, the more disturbances from the irregularities of the agricultural backgrounds, the worse the smoothness of running of the multi-axle block-modular agricultural tractor. The smoothness of the movement depends, to a great deal, on the traction and dynamic indicators, the productivity and agrotechnical qualities of the operation performed, as well as the durability and economical efficiency of the aggregate $[9,10]$. The unsatisfactory smoothness of movement of multi-axle modular agricultural tractors reduces the efficiency of their use in agriculture. Therefore, increasing the smoothness of movement by reducing the mutual vertical oscillations of the power and the technological modules of a multi-axle block-modular agricultural tractor is an urgent problem. There are at least two ways how to improve the smooth running of a multi-axle modular agricultural tractor. The first way is to improve the culture of farming and the quality of its suspension systems. Both directions complement each other. However, it should be noted that even by improving the culture of agriculture, it is impossible to avoid the problem of smooth movement of the block-modular aggregates, since, as it is known, the impact of the disturbance force upon the dynamic system depends on the ratio of the disturbance frequencies and 
the proper vibration frequency of the system. But reconstruction of its own system of the tractor suspension in the classic layout scheme in the direction of creating pneumatic and hydropneumatic suspensions is a complicated and expensive way. The analysis of smooth running of the machine-andtractor aggregates was thoroughly considered in the works by many researchers $[10 ; 11]$, including scientists who have tested the aggregates, based on block-modular power tools for both universal rowcrop and general purposes applications. A possible successful solution is a way to reduce the amplitudefrequency characteristics of the mutual vertical oscillations of the power and the technological modules by creating a dynamic system with viscous damping, which will reduce the specific fuel consumption and increase the technical and economic performance of the aggregates, based on multi-axle modular agricultural tractors. There have been attempts to introduce a hydraulic damper connection between the technological and power module in a horizontal plane [9]. Since the results, obtained by the predecessors, do not make it possible to achieve an increase in the smoothness of the movement of the aggregates, based on multi-axle block-modular agricultural tractors, it became necessary to conduct scientific research, aimed at the solution of this problem. The working hypothesis is that the reduction of mutual vertical oscillations of the power and the technological modules of a multi-axle block-modular agricultural tractor can be achieved by throttling the hydraulic system of its rear suspension mechanism.

The purpose of the work is to study the possibilities of increasing the running smoothness of the multi-axle block-modular tractor by damping its vertical oscillations due to throttling of the hydraulic system of the rear suspension mechanism of the power module.

\section{Materials and methods}

Synthesis of the connection parameters of the modules of a multi-axle block-modular agricultural tractor was carried out by simulating the conditions of its operation on a PC.

By introducing a throttle into the hydraulic system of the rear suspension mechanism of the power module there was created a drosseling system. The technical implementation of the proposed scientific idea is as follows. A multi-axle block-modular agricultural tractor consists of a technological module 1, connected to the power module 2 by means of its rear suspension mechanism, including the central link 3 and the lower link 4, levers and braces 5 . The hydraulic system of the rear suspension mechanism of the power module 2 includes a hydraulic cylinder 6 , the working cavities of which are connected by hydraulic lines 7 and 8 to the hydraulic control valve spool 9, and the drain line 10.

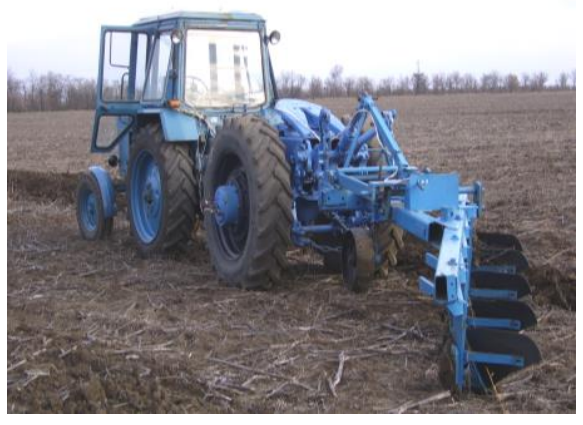

Fig. 1. Ploughing aggregate, based on a multi-axle blockmodular agricultural tractor

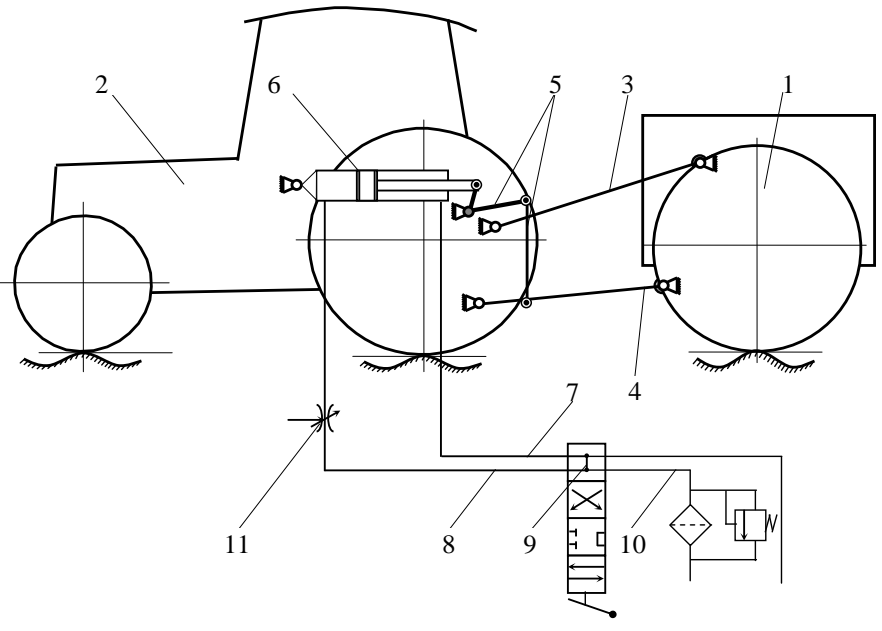

Fig. 2. Diagram of a viscoelastic connection of the power and the technological modules of a multi-axle block-modular agricultural tractor due to throttling of the hydraulic system of the rear suspension mechanism: 1 - technological module; 2 - power module; 3 - central link; 4 - lower links; 5 - levers and braces of the rear suspension mechanism; 6 -hydraulic cylinder; 7,8-hydraulic lines; 9 - hydraulic valve; 10 -drain line; 11 - throttle 
In Fig. 2 a viscous damper (throttle 11) is additionally introduced into the hydraulic system of the power module, which dampens mutual vertical oscillations of the power and the technological modules when the aggregate is moving. The throttle 11 reduces the flow area of the section of the hydraulic line 8 , which connects one of the cavities of the hydraulic cylinder 6 with the valve spool 9. The operation of the main hydraulic cylinder 6, when the hydraulic fluid passes through the calibrated hole of the throttle 11, allows the mechanical energy of oscillations due to viscous friction to be converted into heat energy with its subsequent dissipation in the hydraulic system of the power module. The model of functioning of the hydraulic suspension mechanism of the power module of the multi-axle blockmodular agricultural tractor with a sudden narrowing of the hydraulic line of the main hydraulic cylinder, as a throttling system, was presented by a combination of elastic and dissipative elements (Fig. 3). The system with viscous damping (Fig. 3) consists of an elastic element with stiffness $C_{h}$ and a dissipative element with a resistance coefficient $C_{h}$. When a disturbing oscillatory force $F(t)$ acts upon the system, displacement $Z_{h}(t)$ occurs. In such a case the operation of the hydraulic suspension mechanism of the power module can be presented as an equivalent dynamic system with viscous damping (Fig. 4). Throttling of the hydraulic system of the rear suspension mechanism of the power module determines the case of local pressure losses, called "sudden narrowing" of the hydraulic line.

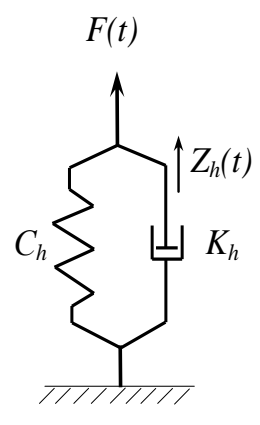

Fig. 3. Linear model with one degree of freedom of an equivalent dynamic system with viscous damping

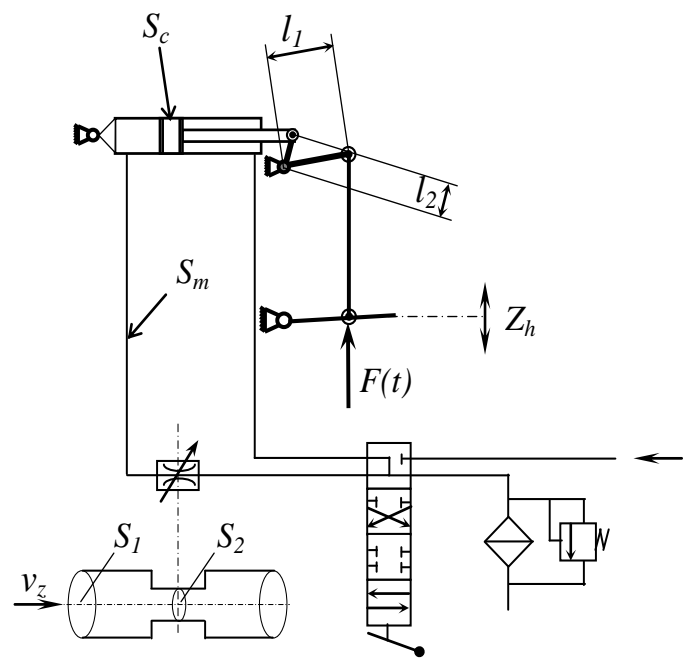

Fig. 4. Diagram of functioning of the hydraulic system of the rear suspension mechanism of the power module as a result of its throttling

The smooth running of a multi-axle block-modular agricultural tractor as part of a ploughing aggregate when creating an elastic-dissipative connection of two modules was experimentally evaluated by recording its vertical accelerations $\ddot{Z}\left(\mathrm{~m} \cdot \mathrm{s}^{-2}\right)$ during the technological process of ploughing. Experimental investigations were carried out under the field conditions on a specially prepared ploughing aggregate in 5 replicates. For this purpose, a tablet computer was used with the Android operating system and built-in accelerometer sensors. Using a special application Accelerometer Meter (version 1.32), digitised output signals of vertical accelerations and their frequency spectrum from accelerometer sensors were recorded over time. The obtained digitalised signals of the vertical acceleration on the tablet computer were imported into the Mathcad software environment to determine their statistical characteristics according to the method described in work [8;12;13].

\section{Results and discussion}

At a sudden narrowing of the hydraulic line of the hydraulic system of the rear suspension mechanism of the energy module, the coefficient of local losses $\zeta$ depends on the ratio of the areas of such narrowing. If we take the cross-sectional area $S_{1}$ as its own (nominal) flow area of the hydraulic line, and the cross-sectional area $S_{2}$ as its changed area due to throttling, then it is possible to construct the dependence of the coefficient of local losses $\zeta$ on the magnitude of throttling $\Omega$ (Fig. 5), which is well approximated analytically $\left(R^{2}=0.9999\right)$ :

$$
\xi=-2 \cdot 10^{-8} \cdot \Omega^{4}+4 \cdot 10^{-6} \cdot \Omega^{3}-3 \cdot 10^{-4} \cdot \Omega^{2}+1.72 \cdot 10^{-2} \cdot \Omega+2 \cdot 10^{-4},
$$


where $\zeta$-coefficient of local pressure losses in the place of sudden narrowing of the flow area of a cylindrical hydraulic line;

$\Omega$ - degree of throttling (\%) of the hydraulic line of the hydraulic system of the power module, numerically equal to

$$
\Omega=\left(1-\frac{S_{2}}{S_{1}}\right) \cdot 100 .
$$

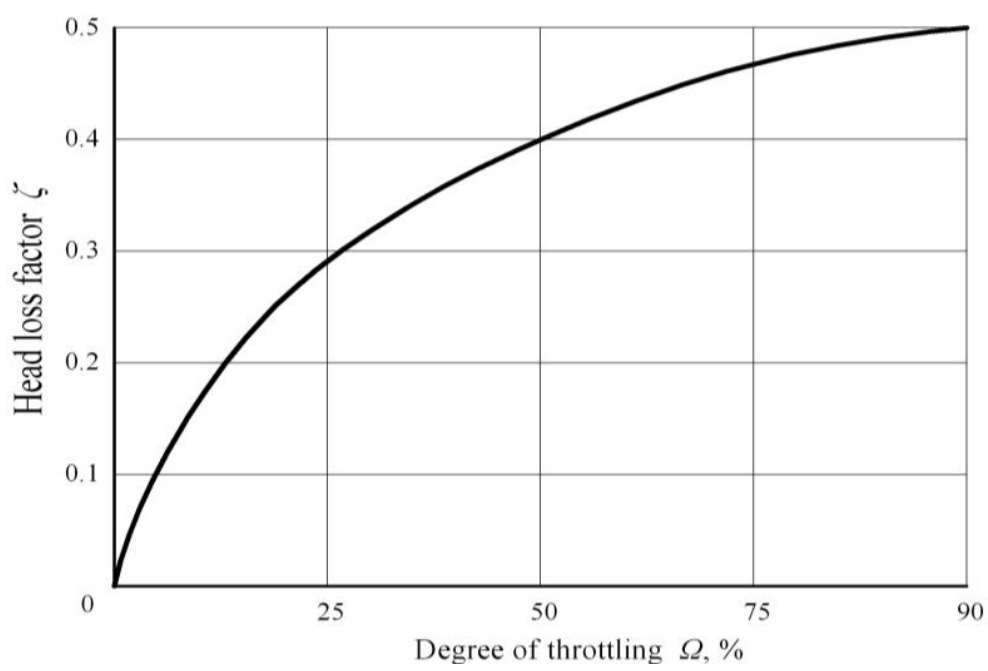

Fig. 5. Dependence of the coefficient of local losses $\zeta$ upon the degree of throttling $\Omega$ of the hydraulic system of the rear suspension mechanism of a multi-axle block-modular agricultural tractor

Analysis of Fig. 5 indicates that with a decrease in the flow area of the hydraulic line of the hydraulic system of the rear suspension mechanism of the power module by $90 \%$ the coefficient of the local pressure losses increases almost 5 times. And this, by the same number of times, allows dissipating the dissipative energy of mutual oscillations of the power and technological modules, using fluid friction. It should be remarked that this relationship is of a nonlinear nature. The performance of deformation of the elastic element of the system is due to the elasticity force:

$$
F_{S}=C_{h} \cdot Z_{h} .
$$

The work performed by the resistance force of the dissipative element of the system (that is, the throttle) is determined by the force:

$$
F_{d}=K_{h} \cdot \dot{Z}_{h} .
$$

The resistance force of the dissipative element of the system converts the mechanical energy of oscillations into thermal energy as a result of viscous friction of the hydraulic fluid in the place of a sudden narrowing of the hydraulic line, with its subsequent dissipation in the hydraulic system:

$$
\grave{A}_{d}=\frac{K_{h} \cdot \dot{Z}_{h}{ }^{2}}{2} .
$$

Local losses of the pressure $h_{m}$ during throttling of the hydraulic system determine the kinetic energy $E_{h}$, which the hydraulic fluid possesses:

$$
\begin{gathered}
h_{m}=\frac{\zeta \cdot v_{z}^{2}}{2 g}, \\
E_{h}=\rho \cdot g \cdot h_{m} \cdot q_{s 1},
\end{gathered}
$$

where $\quad V_{z}$ - fluid flow rate, $\mathrm{m} \cdot \mathrm{s}^{-1}$;

$\rho$ - fluid density, $\mathrm{kg} \cdot \mathrm{m}^{-3}$

$g$ - acceleration of gravity, $\mathrm{m} \cdot \mathrm{s}^{-2}$;

$q_{s 1}$ - flow rate of the hydraulic fluid through the flow area $S_{1}, \mathrm{~m}^{3} \cdot \mathrm{s}^{-1}$ : 


$$
q_{S 1}=S_{m} \cdot V_{Z}
$$

In such a case the coefficient of local losses $\zeta$ of the sudden narrowing of the hydraulic line reveals the essence of the coefficient of resistance of the dissipative forces $K_{h}\left(K_{h} \sim \zeta\right)$. According to Euler's continuity principle about the constancy of the flow rate of an incompressible fluid along the streamline:

$$
V_{C} \cdot S_{C}=V_{Z} \cdot S_{m}
$$

where $S_{C}$ - area of the piston of the hydraulic cylinder, $\mathrm{m}^{2}$;

$V_{C}-$ stroke speed of the main hydraulic cylinder $\left(\mathrm{m} \cdot \mathrm{s}^{-1}\right)$ depends on the speed of the disturbing impact upon the brace of the suspension mechanism of the power module, which is transmitted from the lower link by the coordinate $Z_{h}$ (Fig. 4)

$$
V_{\tilde{N}}=\dot{Z}_{h} \frac{l_{2}}{l_{1}},
$$

where $l_{1}, l_{2}$-design parameters of the hydraulic suspension mechanism of the power module (m), the nature of which is clear from Fig. 4.

After substituting (10) into (9), one can find the value $V_{z}$ :

$$
V_{z}=\dot{Z}_{h} \cdot \frac{S_{C}}{S_{m}} \cdot \frac{l_{2}}{l_{1}} .
$$

Based on the foregoing, it is possible to express the relationship between the value of the dissipative resistance coefficient $K_{h}$ and the speed of disturbing impacts, which are transmitted to the hydraulic suspension mechanism of the power module as a result the mutual vertical oscillations of the two modules:

$$
K_{h}=\dot{Z}_{h} \cdot \zeta \cdot \rho \cdot S_{C} \cdot \frac{l_{2}}{l_{1}} .
$$

Due to the impact of the density of the hydraulic fluid, which, as it is known, depends on the temperature, the rate of the change of the dissipative resistance coefficient $K_{h}$ because of temperature at different degrees of throttling can be expressed by a dependence, shown in Fig. 6.

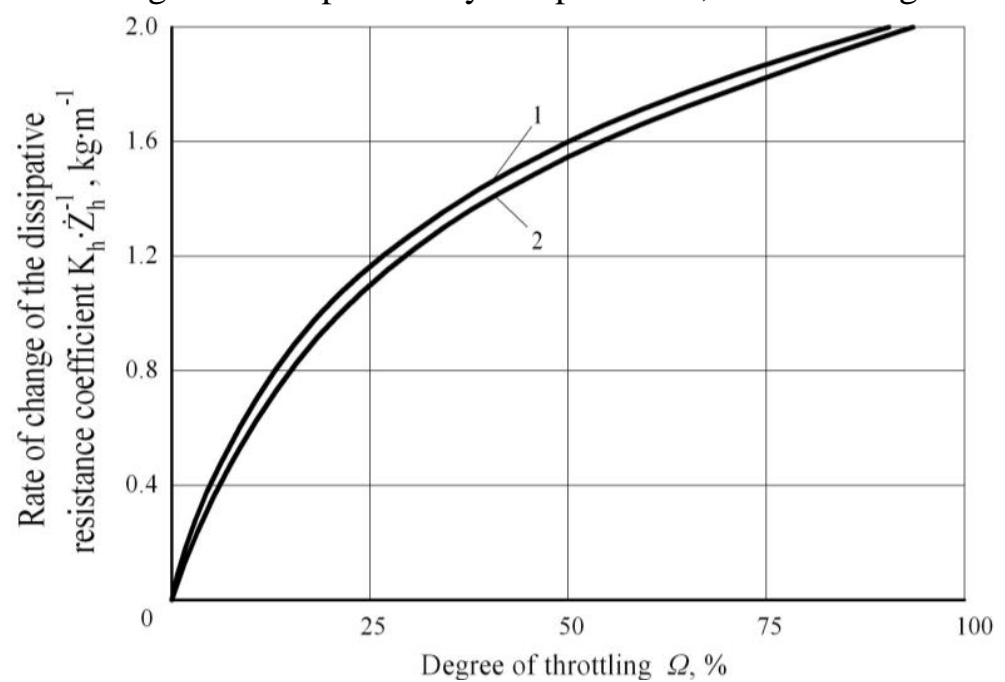

Fig. 6. Dependence of the rate of change of the dissipative resistance coefficient upon the degree of throttling of the hydraulic system at different

temperatures of the hydraulic fluid: $1-20^{\circ} \mathrm{C} ; 2-60^{\circ} \mathrm{C}$

From the analysis of Fig. 6 it can be concluded that heating the hydraulic fluid under the operating conditions will insignificantly affect the quantitative nature of the dependence of the dissipative resistance coefficient $K_{h}$ upon the value of throttling $\Omega$ of the hydraulic system of the power module. This is a positive fact in the efficiency of the proposed method. 
The assessment of the dynamics of vertical oscillations of a multi-axle block-modular agricultural tractor as part of a ploughing aggregate, obtained from the results of the experimental research, confirmed our theoretical investigations. The fact is confirmed that the absence of elastic-dissipative coupling of two modules $\left(K_{h}=0 \mathrm{kN} \cdot \mathrm{s} \cdot \mathrm{m}^{-1}\right)$ is characterised by a very negative oscillatory process in a longitudinal-vertical plane with an unsatisfactory character and magnitude of the spectral density and high dispersion (Fig. 7). At the same time, the increase in the value of the coefficient of resistance of the dissipative coupling of two modules to $K_{h}=1.80$ reduces the frequency range of the basic spectrum of dispersions of accelerations of vertical oscillations to $8 \mathrm{~s}^{-1}$, which is desirable.

The nature of the decrease in the dispersion of acceleration of the vertical oscillations of a multiaxle block-modular agricultural tractor with an increase in the stiffness of the elastic-dissipative connection of its modules is not the same. Intense decrease in the dispersions is observed before the increase in $K_{h}$ to $1.65 \mathrm{kN} \cdot \mathrm{s} \cdot \mathrm{m}^{-1}$, and then it remains approximately constant. The decrease in the dispersion of the accelerations of vertical oscillations of the modular power plant by 3 times is not accidental, since, according to the F-Fisher criterion at a significance level of 0.05 , the null hypothesis of the equality of these statistical estimates is rejected [12].

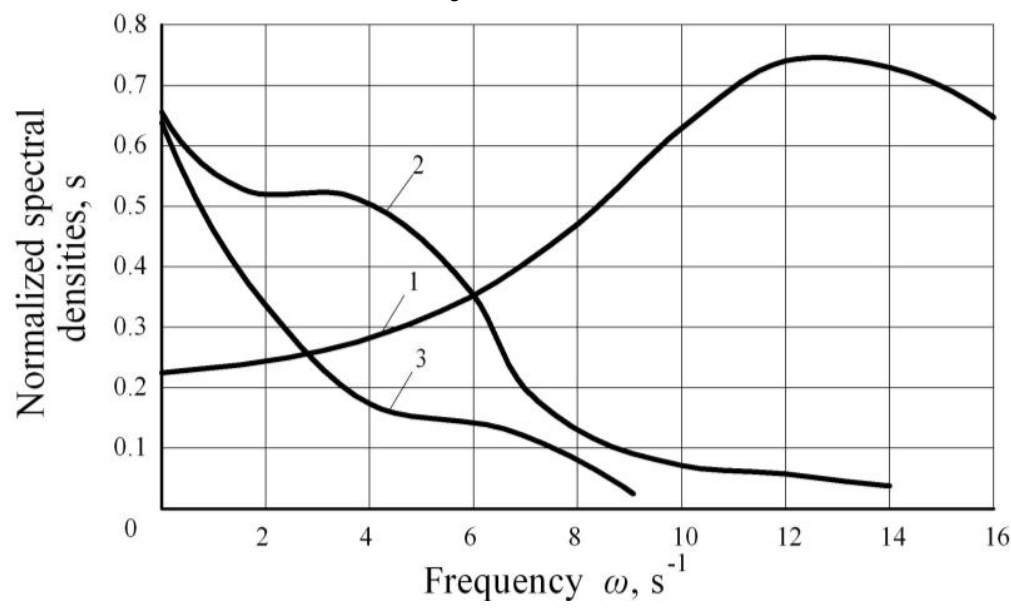

Fig. 7. Normalised spectral densities of acceleration of vertical oscillations of a multi-axle block-modular agricultural tractor at different values of the resistance coefficient of the dissipative connection of the power and technological modules:

$$
1-K_{h}=0 \mathrm{kN} \cdot \mathrm{s} \cdot \mathrm{m}^{-1} ; 2-K_{h}=1.65 \mathrm{kN} \cdot \mathrm{s} \cdot \mathrm{m}^{-1} ; 3-K_{h}=1.8 \mathrm{kN} \cdot \mathrm{s} \cdot \mathrm{m}^{-1}
$$

The results of operational and technological tests of the ploughing aggregate, as part of a modular power plant, showed that the creation of an elastic-dissipative connection of two modules with a drag coefficient of $1.65 \mathrm{kN} \cdot \mathrm{s} \cdot \mathrm{m}^{-1}$ allows increasing its speed by $6 \%$. As a result, the efficiency for 1 hour of the basic operating time of the ploughing aggregate is directly proportional to the increase by the same $6 \%$. In addition to the increase in the efficiency of the aggregate, the specific (per hectare) fuel consumption decreases by an average of $12.4 \%$.

The above material presents a possibility of efficient solution to the problem connected with the smooth movement of block-modular aggregates. Thus, when a multi-axle block-modular agricultural tractor is used in the process of its working movement, creating an elastic-dissipative connection between the power and the technological module by throttling the hydraulic system of the rear suspension mechanism, it is possible to considerably improve the smoothness of its movement. This will essentially improve the performance of aggregates, based on multi-axle modular agricultural tractors, and the quality of the performed technological operation, as well as to ensure the allowed vibration load of the operator.

\section{Conclusions}

1. The resistance coefficient of the viscoelastic connection of modules of a multi-axle block-modular agricultural tractor as a result of throttling of the hydraulic system is directly proportional, but nonlinearly dependent on the coefficient of the local pressure losses of the hydraulic fluid.

2. With an increase in the degree of throttling of the hydraulic system of the rear suspension mechanism of the power module the coefficient of the local pressure losses of the hydraulic fluid 
increases by $90 \%$, which makes it possible to dissipate the dissipative energy of mutual oscillations of the power and the technological modules by means of fluid friction by the same amount.

3. In the range of operating temperatures of the hydraulic fluid $20 \ldots 60{ }^{\circ} \mathrm{C}$, when throttling the hydraulic system of the rear suspension mechanism of the power module under operating conditions, the dissipative resistance coefficient of the elastic-dissipative coupling of two modules of a multi-axle block-modular agricultural tractor changes by no more than 2-5\%. From a practical point of view this increases the reliability of the proposed method for the improvement of the smoothness of its stroke.

4. Creation of a dynamic system with viscoelastic damping by throttling the hydraulic system of the suspension mechanism of the power module allows damping the oscillations of the block-modular aggregates in a vertical plane in a wider frequency band $\left(0 \ldots 16 \mathrm{~s}^{-1}\right)$. At the same time, the dispersion of mutual vertical oscillations of the power and the technological modules during the agricultural operations is reduced more than 3 times, and the allowed vibration load of the operator is ensured. As a result, the efficiency of the ploughing unit increases by 6\%, and the specific (per hectare) fuel consumption decreases by $12.4 \%$.

\section{References}

[1] Viesturs D., Kopiks N. Trends in development of tractor fleet in Latvia. Engineering for rural development, 2017, Vol.16, pp. 534-539.

[2] Vilde A., Rucins A. Simulation of the Impact of the Plough Body Parameters, Soil Properties and Working Modes on the Ploughing Resistance. 10 ${ }^{\text {th }}$ International Conference on Computer Modelling and Simulation, EUROSIM/UKSim2008, Cambridge, 2008, Article number 4489017, pp. 697-702

[3] Nadykto V. Modular energy units. The Ukrainian Farmer. 2010, No 7, pp. 70-71. (In Ukrainian).

[4] Nadykto V., Kyurchev V., Chaplinskyi A. Ways to increase the traction efficiency of modular draft device. IOP Conference Series: Materials Science and Engineering, Vol.1021 (1), 2021, Art. 012043.

[5] Kopiks N., Viesturs D., Valainis O. The Fleet of Tractors on the Farms of Latvia, Its Structure and Energy Intensity. Engineering for Rural Development, Volume 14, 2015, pp. 84-87.

[6] Bulgakov V., Pascuzzi S., Ivanovs S., Nadykto V., Nowak J. Kinematic discrepancy between driving wheels evaluated for a modular traction device. Biosystems Engineering. 2020, Vol. 196, pp. 88-96

[7] Nadykto V. The role of modular power units in the formation of the type of tractors in Ukraine. Tractors and agricultural machinery. No 6, 2010, pp. 22-25. (In Russian).

[8] Ivanovs S., Bulgakov V., Nadykto V., Ihnatiev Ye., Smolinskyi S., Kiernicki Z. Experimental study of the movement controllability of a machine-and-tractor aggregate of the modular type. INMATEH - Agricultural Engineering. Vol. 61 (2), 2020, pp. 9-16.

[9] Sidorov M. Elastic-damping properties of the transport and technological module as part of an agricultural machine-tractor unit. International Research Journal. 2014, No 10-2 (41), pp.119-121. (In Russian).

[10] Sam B., Kathirvel, K. Development and evaluation of vibration isolators for reducing hand transmitted vibration of walking and riding type power tillers. Biosystems Engineering, 2009, 103 (4), pp. 427-437

[11] Yang J., Meng X. Study on vibration mechanism and measures for vibration reducing to the handle of cultivator by virtual prototype technology Nongye Jixie Xuebao/Transactions of the Chinese Society of Agricultural Machinery, 36 (2), 2005, pp. 39-42.

[12] Доспехов Б. Методика полевого опыта (Methodology of field experiments). 2012. Moscow: Nauka, 352 pp. (In Russian)

[13] Веденяпин С.В. Общая методика экспериментальных исследований и обработки опытных данных (General methodology of experimental research and processing of experimental data), Москва: Колос, 1997, 159 p. (In Russian) 